\title{
Bioethics across Cultures - Philosophical and Empirical Justification of the Four Principles of Biomedical Ethics
}

\section{Ebbesen $\mathbf{M}^{*}$}

Department of Culture and Society, Aarhus University, Denmark

*Corresponding author: Ebbesen M, Department of Culture and Society, Aarhus University, Denmark, Tel: +45 28742924; E-mail: MEB@teo.au.dk

Received date: December 09, 2015; Accepted date: January 28, 2016; Published date: January 29, 2016

Copyright: ( 2016, Ebbesen M. This is an open-access article distributed under the terms of the Creative Commons Attribution License, which permits unrestricted use, distribution, and reproduction in any medium, provided the original author and source are credited.

Keywords: Bioethics; Empirical justification; Biomedical ethics

\section{Introduction}

Most research within the academic discipline of bioethics consists of theoretical reflections regarding which ethical theories or principles are useful to analyse ethical issues in the field of biomedicine. The theories of the American ethicists Tom L Beauchamp and James F Childress [1] and the Danish philosophers Jakob Rendtorff and Peter Kemp [2] are examples. Beauchamp and Childress examined considered moral judgements and the way moral beliefs cohere and found that the general principles of beneficence, nonmaleficence, respect for autonomy, and justice play a vital role in biomedical ethics. These authors believe that the four principles are not only specific for biomedical ethics, they are found in all cultures in everyday life because they are part of a cross-cultural common morality shared by all persons committed to morality [1]. The four clusters of principles provide a framework of norms to start with in biomedical ethics. As a starting point no principle is weighted higher than the other principles. When occasion arises, the principles are weighted, balanced, and specified [3].

In this article, I will first shortly present how Beauchamp and Childress justify the four principles philosophically (normatively) and next I will emphasize these authors empirical justification of the principles and give my account of how I think the four principles approach can be developed further by empirical research.

\section{Philosophical - Normative Justification of the Four Principles}

The four principles of beneficence, nonmaleficence, respect for autonomy, and justice are part of a common cross-cultural morality: "The four clusters of principles derive from considered judgements in the common morality and professional traditions in health care, particularly medicine and nursing" [4]. By the term "common morality" Beauchamp refers to "the set of norms shared by all persons committed to the objectives of morality. The objectives of morality, I will argue, are those of promoting human flourishing by counteracting conditions that cause the quality of people's lives to worsen". "It is applicable to all persons in all places, and all human conduct is rightly judged by its standards. Virtually all people in all cultures grow up with an understanding of the basic demands that morality makes upon everyone. They know not to lie, steal, break promises, and the like" [5]. Beauchamp \& Childress write that since many amoral persons do not care about the moral obligations and demands, it would be ridiculous to suppose that these common morality norms are accepted by all persons. Nonetheless, they believe that all persons in all cultures who take moral conduct seriously do accept the norms of the common morality. The common morality plays a foundational role in the latest editions of Beauchamp and Childress' theory. They write that no more basic moral content exists than the body of rules and judgements developed from the four clusters of principles. Furthermore, they state that the common morality can function as a basis for evaluation of groups whose moral conduct seems in some respect deficient [1].

\section{Empirical Justification of the Four Principles}

Beauchamp and Childress not only justify the four principles normatively in relation to the common morality, they also justify them empirically, arguing that the common morality describes what all morally serious people believe [6]. Without presenting any data, they claim that the usefulness of the four principles of beneficence, nonmaleficence, respect for autonomy, and justice in biomedical ethics can be tested empirically, and Beauchamp requests the design of a qualitative empirical research project to investigate the question $[1,5]$. However, not much empirical research has been done to explore ethical issues in biomedical settings in general, but there are few examples [7-13] and apparently, only one empirical study has specifically investigated the importance of the four principles, this is a study conducted by Ebbesen et al., [14-17].

Most qualitative empirical research in the field of biomedical ethics concerns the ethical reasoning of physicians and nurses [7-12]. Some researchers $[8,9,11]$ have studied the differences in ethical reasoning between physicians and nurses, men and women. Other researchers $[7,10]$ have investigated how physicians handle situations where there is a tension between the obligation to respect the patients' right to autonomy and the obligation to promote their health. However, little is known about the differences in ethical considerations at stake between physician oncologists working in different cultural settings.

Beauchamp and Childress are inspired by the philosopher W. D. Ross with regard to their choice of principles. Ross regards the duties of fidelity, reparation, gratitude, justice, beneficence, selfimprovement, and nonmaleficence as prima facie duties [18]. These prima facie duties are objective facts involved in the nature of situations in human practice, therefore they are self-evident and do not need to be proven [18]. Beauchamp and Childress' choice of principles is almost in agreement with Ross' prima facie duties. However, ethical considerations unrecognized by Beauchamp \& Childress' theory may be brought to light by empirical studies of the ethical reasoning of health care professionals.

\section{A Danish Empirical Study Exploring the Importance of the Four Principles}

The findings of Ebbesen et al., [14-16] show that Danish physician oncologists and biomedical researchers use the four principles in their 
daily work, however, no empirical results exist telling whether the principles are applicable to other cultural settings [19]. Here, I will first present the study of Ebbesen et al., [14-16] investigating health care professionals and biomedical researchers in Danish setting and thereafter I will sketch the future perspectives of the study extending it to other cultures.

The aim of this Danish study was (1) to develop a method suitable for empirical investigation of the ethical reasoning within biomedicine [17] and (2) to use this method to test whether there is a difference in the ethical considerations or principles at stake between Danish physician oncologists and molecular biologists [14-16].

This study was based on 12 semi-structured interviews with three groups of respondents: Two groups of molecular biologists conducting basic research at either a public university or a private biopharmaceutical company, and one group of physician oncologists working in the clinic at a public hospital. The decisive criterion for sample size is the point where variation ceases; saturation tends to occur when the number of interviews reaches around $15 \pm 10[20,21]$. We observed that data saturation was beginning to appear after interviewing 9 respondents. The reason to select these three groups of respondents was to test whether ethical problems about human beings are perceived as more distant for molecular biologists employed at the university conducting pure basic research than for physicians working in a doctor-patient relationship. Molecular biologists employed in a private biopharmaceutical company possess a 'middle position' between conducting pure basic research and working with patients, they conduct basic research with the aim of developing pharmaceuticals to patients.

The method used is thoroughly described in Ebbesen \& Pedersen [17], but I will shortly review it below. The method is based on the phenomenological hermeneutical method of Lindseth \& Norberg [22], which is developed to reveal the ethical thinking of physicians and nurses. Lindseth and Norberg [22] are inspired by philosopher E. Husserls descriptive phenomenology in as much as the aim is to describe the lived experience of the interviewees. It is essential that the researcher has a phenomenological attitude, hence sheds all prior personal knowledge to grasp the essential lived experiences of the respondents [23]. The approach is hermeneutical since the task is to understand the experiences expressed in the interview texts. Hermeneutics goes beyond the description of core concepts and essences to look for meanings embedded in the life practices. These meanings are not always apparent to the respondents but can be gleaned from the narratives (the interview texts) they produce [23]. The phenomenological hermeneutical approach was used both for the design of the interview guide and for data analysis.

The ethical reasoning of physicians and molecular biologists was explored by use of semi-structured interviews $[21,24]$. The interview guide used consists of 13 main questions, each containing a number of sub-questions. The single interview lasted for 1 hour and 5 minutes in average and the interview text was transcribed word-for-word. Please find a detailed description of the theory behind the interview questions in Ebbesen and Pedersen [17].

The data were analysed using a phenomenological hermeneutical method for interpreting interview texts inspired by the theory of interpretation presented by Ricoeur $[17,22,25]$. In the following the three steps of data analysis are briefly described.

\section{Naïve reading}

The text is read several times in order to grasp its meaning as a whole. The interpreter tries to read the text with a phenomenological attitude, so as to be open enough to allow the text to speak to him/her. The naïve reading is regarded as a first conjecture and it has to be validated or invalidated by the subsequent structural analysis $[17,25]$.

\section{Structural analysis}

According to Ricoeur to understand a text is to follow its movement from what it says to what it talks about $[17,25]$. In the structural analysis we move from what the text says to what it talks about, first by describing units of meaning (what is said) and next by identifying and formulating units of significance (what is talked about) and themes $[17,25]$. First, the whole text is read and divided into units of meaning (what is said). Secondly, the units of meaning are reflected on in relation to the naïve understanding. Then the units of meaning are sorted and condensed and units of significance are formulated (what is talked about). Next, units of significance are condensed even more and themes are formulated $[17,25]$. The themes are reflected on against the background of the naïve understanding to see whether the themes validate or invalidate the naïve understanding. If the structural analysis invalidates the naïve understanding, the whole text is read again and a new naïve understanding is formulated and checked by a new structural analysis. This process of comparing the naïve reading and the structural analysis is repeated until the naïve understanding is validated through the structural analysis $[17,25]$.

\section{Critical interpretation}

The text is read again as a whole with the naïve understanding and the validated themes in mind and with an as open mind as possible. The themes are then reflected on in relation to the literature which can help to revise, widen, and deepen our understanding of the text. However, since this was a phenomenological hermeneutical study we did not force the literature perspective on the interview text but let the literature illuminate the interview text and the interview text illuminate the literature $[17,24,25]$.

We [14-16] found that physician oncologists and molecular biologists employed in a private biopharmaceutical company have the specific principle of beneficence in mind in their daily work. Both groups are motivated to help sick patients. According to the study, molecular biologists explicitly consider nonmaleficence in relation to the environment, the researchers health, and animal models; and only implicitly in relation to patients or human subjects. In contrast, considerations of nonmaleficence by physician oncologists relate to patients or human subjects. Physicians and molecular biologists both consider the principle of respect for autonomy as a negative obligation in the sense that the informed consent of patients should be respected. However, in contrast to molecular biologists, physicians experience the principle of respect for autonomy as a positive obligation as the physician offers a medical prognosis based upon the patients' wishes and ideas, mutual understanding, and respect. Lastly, this study discloses utilitarian characteristics in the overall concept of justice proposed by physician oncologists and molecular biologists from the private biopharmaceutical company. Molecular biologists employed at a public university reflect about allocation, however, they do not propose a specific theory of justice. Hence, this study [14-16] demonstrates that the four bioethical principles of respect for autonomy, beneficence, nonmaleficence, and justice proposed by 
Beauchamp and Childress are reflected in the daily work of Danish physicians and molecular biologists and applicable to the Danish biomedical setting.

\section{Future Perspectives to Further Development the Four Principles Approach based on Empirical Research}

Beauchamp and Childress [5,6] claim that their bioethical principles are part of a common cross-cultural morality. However, some of their critics [26] state that the principle based theory is developed from American common morality and that it mirrors certain aspects of American society. And for this reason alone it might be untransferable to other contexts and other societies. More scholars have made theoretical attempts claiming that the four principles are not useful in other cultural setting than the American [27]. Nonetheless, the findings of the project by Ebbesen et al., [14-16] indicate that the principles are transferable to Danish biomedical practice. Future perspectives of the study by Ebbesen et al., [14-17] are to investigate biomedical practices in different cultures such as Scandinavian, Southern European, Asian, and American cultures to test whether Beauchamp and Childress' principles are cross-cultural and thereby have a universal perspective. Below I will sketch a setup of an empirical project which could investigate whether there is a difference in the ethical considerations or principles at stake between physician oncologists working in different cultural settings such as Scandinavian, Southern European, Asian, American cultures [19].

This study will be based on 16 semi-structured interviews. We have four groups of respondents: 4 physician oncologists working in the clinic at a public hospital in Denmark, Italy, China, and USA, respectively. The interview guide and methods are similar to the ones used in the project in the Danish context by Ebbesen et al. [14-17] as described above. And, the aim, methods, data analysis, and literature review are described more detailed in Ebbesen et al., [19].

\section{Conclusion}

In this article I have first examined how Beauchamp and Childress justify the four principles approach normatively by the common morality. Next, I have explored how to justify this approach empirically by qualitative empirical studies. I have presented a Danish study as an example. Furthermore, I have given suggestions to how to investigate whether there are indications that the bioethical principles of Beauchamp and Childress are specifically western or whether they are cross cultural. Despite the fact that critics indicate that the principles are unsuited for Europe and East Asia, Beauchamp maintains that empirical research can be used to test the hypothesis that a common cross cultural morality based on the four principles does exist or not. I argued that indications for a common morality can be explored by qualitative research based on narrative interviews. I outlined a phenomenological-hermeneutical method which we have already used to investigate the ethics of Danish oncologists and molecular biologists. And I argued that this method is also useful to investigate the ethics of oncologists in North and South European, East Asian, and American cultures [19].

\section{References}

1. Beauchamp TL, Childress JF (2013) Principles of Biomedical Ethics. 7th ed. Oxford University Press, Oxford, UK
2. Rendtorff J, Kemp P (2002) Basic ethical principles in European bioethics and biolaw. Vol. 1: autonomy, dignity, integrity and vulnerability. Medicine, Health Care and Philosophy 5: 235-244.

3. Beauchamp TL (2003) Methods and principles in biomedical ethics. J Med Ethics 29: 269-274.

4. Beauchamp TL, Childress JF (2009) Principles of Biomedical Ethics. 6th ed. Oxford University Press, Oxford, UK.

5. Beauchamp TL (2003) A Defense of the Common Morality. Kennedy Inst Eth J 13: 259-274.

6. Beauchamp TL, Childress JF (2001) Principles of Biomedical Ethics. 5th ed. Oxford University Press, Oxford, UK.

7. Rossel P (1986) Empirical ethics â€“ its necessity and method. (In Danish). Philosophia 1986 14: 90-103.

8. Uden G, Norberg A, Lindseth A, Marhaug V (1992) Ethical reasoning in nurses' and physicians' stories about care episodes. J Adv Nurs 17: 1028-1034.

9. Lindseth A, Marhaug V, Norberg A, Uden G (1994) Registered nurses' and physicians' reflections on their narratives about ethically difficult care episodes. J Adv Nurs 20: 245-250.

10. Bremberg S, Nilstun T (2000) Patients' autonomy and medical benefit: ethical reasoning among GPs. Fam Pract 17: 124-128.

11. Sørlie V, Lindseth A, Udén G, Norberg A (2000) Women physicians' narratives about being in ethically difficult care situations in paediatrics. Nurs Ethics 7: 47-62.

12. Soerlie V, Foerde R, Lindseth A, Norberg A (2001) Male physicians' narratives about being in ethically difficult care situations in paediatrics. Soc Sci Med 53: 657-667.

13. van Thiel GJ, van Delden JJ (2001) The principle of respect for autonomy in the care of nursing home residents. Nurs Ethics 8: 419-431.

14. Ebbesen M, Pedersen BD (2008) The principle of respect for autonomy-concordant with the experience of oncology physicians and molecular biologists in their daily work? BMC Med Ethics 9: 5 .

15. Ebbesen M, Pedersen BD (2008) The Role of Ethics in the Daily Work of Oncology Physicians and Molecular Biologists - results of an empirical Study. Business and Professional Ethics Journal 27: 75-101.

16. Ebbesen M, Pedersen BD (2007) Empirical investigation of the ethical reasoning of physicians and molecular biologists - the importance of the four principles of biomedical ethics. Philos Ethics Humanit Med 2: 23.

17. Ebbesen M, Pedersen, BD (2007) Using Empirical Research to Formulate Normative Ethical Principles in Biomedicine". Med Health Care Philos 10: 33-48.

18. Ross WD () The right and the good. Oxford: The Clarendon Press, 1930.

19. Ebbesen M, Andersen S, Pedersen BD (2012) Further Development of Beauchamp and Childress' Theory Based on Empirical Ethics. J Clin Res Bioeth S6: e001: 1-7.

20. Morse J (1993) Drowning in Data. Qualitative Health Research 1993, 3:267-69.

21. Kvale S (1996) Interviews: An Introduction to Qualitative Research Interviewing. USA: Sage, Thousand Oaks.

22. Lindseth A, Norberg AA (2004) Phenomenological Hermeneutical Method for Researching Lived Experience. Scand J Caring Sci 18: 145-153.

23. Lopez KA, Willis DG (2004) Descriptive Versus Interpretive Phenomenology: Their Contributions to Nursing Knowledge. Qual Health Res 14: 726-735.

24. Kvale S (1983) The Qualitative Research Interview: a Phenomenological and a Hermeneutical mode of Understanding. Journal of Phenomenological Psychology 14: 171-196.

25. Pedersen BD (1999) Nursing Practice, Language and Cognition. Ph.D.Thesis. University of Aarhus, Denmark.

26. Holm S (1995) Not just autonomy--the principles of American biomedical ethics. J Med Ethics 21: 332-338.

27. Gillon R (ed.) (1994) Principles of Health Care Ethics. Chichester: Wiley. 